Kansas State University Libraries

New Prairie Press

\title{
Editor's Preface, Table of Contents, and List of Attendees
}

George A. Milliken

Follow this and additional works at: https://newprairiepress.org/agstatconference

Part of the Agriculture Commons, and the Applied Statistics Commons

\section{(c) $(1) \ominus$}

This work is licensed under a Creative Commons Attribution-Noncommercial-No Derivative Works 4.0 License.

\section{Recommended Citation}

Milliken, George A. (1992). "Editor's Preface, Table of Contents, and List of Attendees," Conference on Applied Statistics in Agriculture. https://doi.org/10.4148/2475-7772.1389

This is brought to you for free and open access by the Conferences at New Prairie Press. It has been accepted for inclusion in Conference on Applied Statistics in Agriculture by an authorized administrator of New Prairie Press. For more information, please contact cads@k-state.edu. 


\section{APPLIED \\ STATISTICS IN \\ AGRICULTURE}

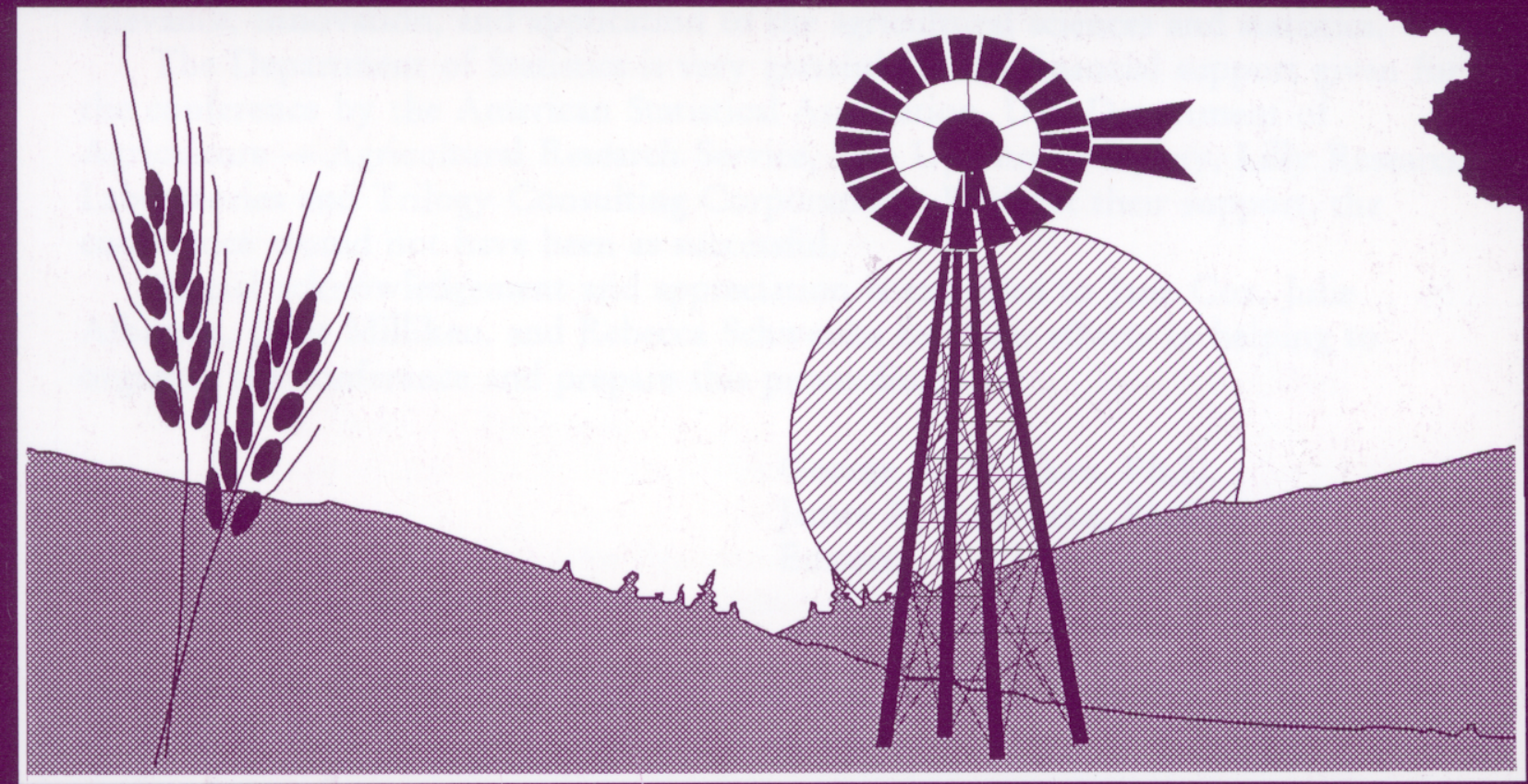

Proceedings of the 1992 Kansas State University Conference on Applied Statistics in Agriculture

Sponsored by:

Department of Statistics

Kansas State University

Manhattan, Kansas 66506

April 26-28, 1992 


\section{Preface}

This proceedings contains papers presented at the fourth annual Kansas State University Conference on Applied Statistics in Agriculture, held in Manhattan, Kansas, April 26 through 28, 1992. The purpose of the conference is to provide a forum for discussion on the application of statistics to problcins in agricultural sciences. The papers presented at the conference and included in this proceedings are intended to provide information to both the statistician and agricultural researcher on current issues of agricultural and general statistical interest.

The keynote speaker was J. Stuart Hunter, Ph.D.

Each contributed paper was refereed before being accepted for publication in this proceedings. Papers were accepted for publication based on their interest, relevance, innovation, and application to the agricultural sciences and statistics.

The Department of Statistics is very grateful for the financial support given for the conference by the American Statistical Association, U.S. Department of Agriculture - Agricultural Research Service, The Upjohn Company, Lilly Research Laboratories and Trilogy Consulting Corporation. Without their support, the conference would not have been as successful.

Special acknowledgement and appreciation is extended to Jane Cox, Julie Albrecht, Janet Milliken, and Rebecca Schwenke for their efforts in helping to organize the conference and prepare this proceedings.

George A. Milliken, Ph.D.

James R. Schwenke, Ph.D.

Editors 


\section{Table of Contents}

Beyond Linearity and Independence

J. Stuart Hunter . . . . . . . . . . . . . . . . . . 1

Co-Effect Analysis of Variance: A New Method for Unbalanced Data

André Plante . . . . . . . . . . . . . . . . . . . . . . 15

Studying Herbicide Resistance Using Treatment Area Dynaninics Model

Agam N. Sinha, Dale L. Shaner . . . . . . . . . . . . . . 27

Prevalence Rate Differences Based on Herdmate Comparisons

Jerome M. Sacks, Randall C. Cutlip,

Amy L. Weaver and Howard D. Lehmkuhl . . . . . . . . . . 41

Utilization of the Line-Intercept Method to Estimate the Coverage, Density,

and Average Length of Row Skips in Cotton and Other Row Crops

Jeffrey L. Willers, Sreenivasa R. Yatham,

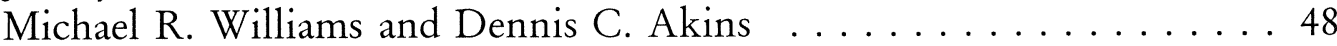

Statistical Analysis of Genotype-by-Environment Interaction Using the AMMI

Model and Stability Estimates

Bahman Shafii and William J. Price $\ldots \ldots \ldots \ldots \ldots \ldots \ldots$

A Markov Chain Model to Assess Resistance of Cattle to Horn Flies

Edward Gbur and C. Dayton Steelman . . . . . . . . . . . . 73

Confidence Intervals for Soil Properties Based on Differing Statistical

Assumptions

Fred J. Young, R. David Hammer and Jon M. Maatta . . . . . . . 87

Designed Experiments in the Presence of Spatial Correlation

David B. Marx . . . . . . . . . . . . . . . . . . . . . . . . . . . . . 104

A Simple Alternative to the Standard Statistical Model for the Analysis of Field

Experiments with Latin Square Designs

C. Philip Cox and Jeff B. Meeker . . . . . . . . . . . . . . 125

Planning a Safety Study of an Agricultural Product: Effects of Land

Application of Phosphogypsum on Random Flux

Ramon C. Littell and Sudeep Kundu . . . . . . . . . . . . . . . . 140

Estimating Variance Functions for Weighted Linear Regression

Michael S. Williams, Hans T. Schreuder,

Timothy G. Gregoire and William A. Bechtold ............. 153 
The Analysis of Tree Ring Chronologies Using a Mixed Linear Model

O. Brian Allen, Daniel A.J. Ryan and David L. McLaughlin ....... 161

Options for Analyzing Unbalanced Split-Plot Experiments: A Case Study

Marta D. Remmenga and Dallas E. Johnson . . . . . . . . . . . 170

Analysis of Mixed Models Without Mixed Models Software

George A. Milliken . . . . . . . . . . . . . . . . . . . 180

Analyzing Split-Plot and Repeated-Measures Designs Using Mixed Models

Russ Wolfinger, Nancy Miles-McDermott and Jenny Kendall .... . 190

When Should Random Effects be Included in Estimable Functions and When

They Should Not?

David C. Blouin . . . . . . . . . . . . . . . . . . . . . . 201

An Example of Path Analysis Applied to Classification Variables

Richard E. Lund and Albert L. Scharen . . . . . . . . . . . 213

Regression Modeling Using Principal Components

Shahar Boneh and Gonzalo R Mendieta . . . . . . . . . . . 222

Soil Properties and Landtypes - Classification and Identification with

Discrimination Analysis

R. David Hammer and John W. Philpot . . . . . . . . . 233

Simplified Data Analysis for Generally Balanced but Messy Experimental

Designs

Richard E. Lund . . . . . . . . . . . . . . . . . . . . 243

A Comparison of Double Sampling Regression Estimators

Dennis L. Clason and G. Morris Southward . . . . . . . . . . 257

The Need for the Analysis of Treatment x Period Interaction in Animal

Experiments

L.A. Goonewardene and L.Z. Florence . . . . . . . . . . . 265

List of Conference Attendees . . . . . . . . . . . . . . . . 280 


\section{List of Conference Attendees}

Adnan, Arisman, Kansas State University Al-Gabir, Khalid, Kansas State University Al-Shiha, Abdullah, Kansas State University Al-Zaid, Munther, Kansas State University Allen, Fred, University of Tennessee Allen, O. Brian, University of Guelph Ballou, Lynda, Kansas State University Barbec, Mark, Iowa State University Blouin, David, Louisiana State University Boldman, Keith, University of Tennessee Boucher, Joseph F., The Upjohn Company Boyer, John, Kansas State University Boykin, Debbie, USDA-ARS

Brown, Michael, USDA, ARS

Camp, Mary, USDA/ARS

Campbell, Larry G., USDA/ARS

Chang, Kuo-Hsing, Kansas State University

Chartier, Kevin, Kansas State University

Chen, Guang, Kansas State University

Chen, Shaohsin, Kansas State University

Chester, S. Theodore, The Upjohn Company

Chew, Victor, University of Florida

Choi, Jaesung, Kansas State University

Clason, Dennis, New Mexico State University

Claypool, P. Larry, Oklahoma State University

Cornelius, Paul, University of Kentucky

Cox, C. Philip, Iowa State University

Cox, David F., Iowa State University

Darrow, Patrick, Imperial Holly Corporation

Deluyker, Hubert, The Upjohn Company

Dickinson, Jennifer, Kansas State University

Dong, Jian, Kansas State University

Douglass, Larry W., University of Maryland

Evenson, Paul, South Dakota State Univ.

Fergen, Brian, Kansas State University

Feyerherm, Arlin M., Kansas State University

Freese, Larry D., USDA

Fryer, Holly, Kansas State University

Gbur, Edward, University of Arkansas

Goad, Carla, Kansas State University

Goonwardene, Laki, Alberta Agriculture

Gregoire, Timothy G., Virginia Polytechnic Inst. \& State Univ.

Hadarbach, Driss, University of Nebraska

Hammer, R. David, University of MissouriRolla

Hanford, Kathryn, Harris Laboratories Hanson, Boyd J., The Upjohn Company

Harder, John, Kansas State University Higgins, Jim, Kansas State University
Hinz, Paul N., Iowa State University Howell, Kim, Kansas State University $\mathrm{Hu}$, Xiaoli, Utah State University Hussain, Ghulam, Kansas State University Isbell, Molly, Iowa State University Jansonius, Le Wayne, USDA-FCIC Johnson, Dallas E., Kansas State University Jui, Perry Y., Agriculture Canada Kachman, Stephen D., The University of Nebraska-Lincoln

Keighley, John, Kansas State University Kemp, Ken, Kansas State University Kendall, Jenny, SAS Institute Khamis, Imad, Kansas State University Kiernan, Kathleen, Kansas State University Knapp, Brad, USDA, ARS, LARRS Kratzer, D. Dal, The Upjohn Company Krause, Gary F., University of MissouriColumbia

Ladurini, Dan, Trilogy Consulting Corporation Lancaster, Vicki, Kansas State University Lee, Ming Hsiang, University of NebraskaLincoln

Lecdle, Jane, Kansas State University Lewis, Jerry W., Marion Merrell Dow Inc. Littell, Ramon C., University of Florida Loughin, Thomas, Iowa State University Louv, William C., Marion Merrell Dow, Inc.

Lu, Biao, Kansas State University

Lumpe, Torrey, Flint Hills Statistical Consulting

Lund, Richard, Montana State University

Luo, Xianwen, Kansas State University

Mackey, Bruce, USDA/ARS

Martinez, Ricardo, Iowa State University

Marx, David, The University of NebraskaLincoln

Matekaitis, Leslie, DeKalb Plant Genetics

Mayer, Hugo E., Trac

McDermott, Nancy, SAS Institute

McPherson, Iris, Oklahoma State University

McVey, Anita, Iowa State University

Milliken, George A., Kansas State University

Milliken-Janne, April, University of NebraskaLincoln

Moore, Terri, Kansas State University

Morton, Lisa, Pioneer Hi-Bred

Mowrey, Daniel H., Eli Lilly \& Company

Mustofa, Kansas State University

Nassar, Raj F., Kansas State University 
Neill, Jim, Kansas State University

Nelsen, Terry, USDA-ARS

Nelson, Paul, Kansas State University

Njuho, Peter, Kansas State University

Noble, Joyce, Kansas State University

Noble, William, Kansas State University

Noren, Win, Kansas State University

Novotny, John, U.S. Navy

Ntabana, Jean Bosco, University of NebraskaLincoln

Nyquist, Wyman E., Purdue University

O’Brien, Timothy E., North Carolina State Univ.

Odejar, Maria, Kansas State University

Olmsted, Ann, Syntex

Ordoobadi, Sharon, Kansas State University

Palmquist, Debra, USDA/ARS

Parvin-Nejad, Habib, Kansas State University

Payton, Mark, Oklahoma State University

Peng, Xiaojun, Kansas State University

Perng, Peter, Kansas State University

Peterson, Del Var, Texas A \& M University

Phillips, John G., USDA-ARS

Pirbhai, Erfan, University of Nebraska-Lincoln

Plachecki, JoAnn, Kansas State University

Plante, André, Université du Québec à Montréal

Popham, Thomas, USDA/ARS

Pousti, Mehdi, Kansas State University

Pozin, Yael, Ministry of Agriculture

Reid, William, Pecan Experiment Field

Remmenga, Marta, Kansas State University

Renganathan, Mala, University of NebraskaLincoln

Rhaesa, Beth, Kansas State University

Richardson, Gary, Colorado State University

Rolka, Debby, Kansas State University

Rolka, Henry, Kansas State University

Rust, Amber, University of Nebraska-Lincoln

Sacks, Jerome, USDA ARS

Sanders, William R., BMDP Statistical Software, Inc.

Schreiber, Elizabeth, Kansas State University

Schwenke, Jim, Kansas State University

Scolari, Kristine, Kansas State University

Scott, Jeff, USDA Forest Service

Shafii, Bahman, University of Idaho

Sinha, Agam N., American Cyanamid Company

Smothers, Clark, Pfizer

Ssekiboobo, Agnes M.N., Makerere University

Stroup, Walter, The University of NebraskaLincoln
Taylor, Veronica, Kansas State University Tonkinson, Lealon, Eli Lilly \& Company Vinyard, Bryan, USDA/ARS/SRRC

Weeks, David, Oklahoma State University Wei, Greg, Marion Merrell Dow Inc.

Whitehand, Linda, USDA

Wilcox, C.J., University of Florida

Willers, Jeffrey, USDA-ARS

Williams, Michael, USDA Forest Service

Wilson, Karen, Kansas State Univesity

Wilson, Mary, Kansas State University

Wilson, Ruel, USDA, ARS, SAA

Wolfinger, Russ, SAS Institute, Inc.

Wu Green, Lie-Ling, Iowa State University Yang, Winston, Kansas State University Young, Fred, University of Missouri-Rolla Young, Linda, The University of NebraskaLincoln 